Cross-sectional SPMs of regional FA reductions superimposed onto sagittal and axial FA maps: Left, significantly reduced FA in FTD or AD compared to $\mathrm{CN}$. Right, significant FA reductions in FTD compared to $\mathrm{AD}(\mathrm{AD}>\mathrm{FTD})$
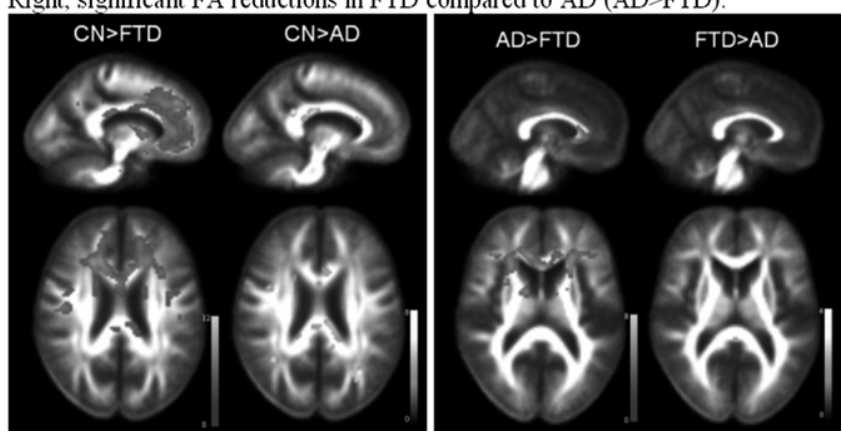

P1-311

USE OF THE SUPPORT VECTOR MACHINE AND SENSITIVITY OF AN ALZHEIMER'S DISEASE-RELATED REGION-OF-INTEREST GRAY MATTER CLASSIFIER IN IDENTIFYING AMNESTIC MILD COGNITIVE IMPAIRMENT SUBJECTS WHO CONVERT TO ALZHEIMER'S DISEASE: PRELIMINARY FINDINGS FROM THE ALZHEIMER'S DISEASE NEUROIMAGING INITIATIVE

Hang Zhang ${ }^{1}$, Tong $\mathrm{Wu}^{1}$, Min-Hyeok Bae ${ }^{1}$, Eric M. Reiman ${ }^{2}$, Gene E. Alexander ${ }^{3}$, Paul M. Thompson ${ }^{4}$, Clifford R. Jack Jr. ${ }^{5}$, Kewei Chen ${ }^{2},{ }^{1}$ Arizona State University, Tempe, AZ, USA; ${ }^{2}$ Banner Alzheimer's Institute, Phoenix, AZ, USA; ${ }^{3}$ University of Arizona, Tucson, AZ, USA; ${ }^{4}$ University of California, Los Angeles, Los Angeles, CA, USA; ${ }^{5}$ Mayo Clinic and Foundation, Rochester, MN, USA. Contact email: kchen@math.la.asu.edu

Background: Structural magnetic resonance imaging (MRI) has been used to distinguish Alzheimer's disease (AD) patients from normal controls (NC) and predict conversion from amnestic mild cognitive impairment (MCI) to probable AD. Data from the multi-center Alzheimer's Disease Neuroimaging Initiative (ADNI) give researchers the chance to compare different voxel-based and region-of-interest (ROI) image-analysis strategies in terms of their ability to address these questions and others related to the early detection and tracking of AD. Our objectives were to use the SPM5 segmented gray matter MRIs, Automated Anatomic Labeling (AAL) ROIs and support vector machine (SVM) to generate a classifier to distinguish AD cases from $\mathrm{NC}$ and test its sensitivity in identifying amnestic MCI subjects who convert to $\mathrm{AD}$ at 6 months. If it performed properly, one would expect the algorithm to classify converter MCI subjects as AD rather than as NC. Methods: ADNI MRI images were downloaded from 59 probable AD patients and $59 \mathrm{NC}$ and from 18 amnestic MCI patients who converted to probable AD six months later. SPM5 was used to generate modulated gray matter (GM) maps in template space and characterize gray matter measurements from 116 AAL-derived ROIs. SVM was then applied to these regional data using the radial basis function as a kernel and five-fold cross-validation to optimize the distinction between probable AD patients and NC. Finally, the AD-related SVM classifier was used to evaluate its accuracy in classifying MCI who converted at 6-months with AD subjects. Results: Our ROI-based SVM strategy distinguished AD from NC with an accuracy of $83 \pm 1 \%$. This resulting AD-related SVM classifier correctly identified 14 of the 18 MCI patients who converted to probable AD in six months, suggesting a sensitivity of 78\%. Conclusions: Use of SVM, ROIs and an AD-related classifier has promise in predicting conversion from amnestic MCI to AD. Additional analyses are needed to determine the extent to which this classification strategy, voxel-based SVM strategies that do not rely on AAL or other ROIs, and other data analysis methods are both sensitive and specific in predicting rates of conversion from amnestic MCI to AD over 6 months and longer time-frames.
P1-312 DIFFERENTIATING AMNESTIC MILD COGNITIVE IMPAIRMENT CONVERTING TO PROBABLE ALZHEIMER'S DISEASE FROM STABLE AMINESTIC MILD COGNITIVE IMPAIRMENT USING FDG-PET AND AN ALZHEIMER'S DISEASE-RELATED HYPOMETABOLISM OVERLAP INDEX

Napatkamon Ayutyanont ${ }^{1,2}$, Kewei Chen ${ }^{1,2}$, Xiaofen $\mathrm{Liu}^{1,2}$, Cole Reschke ${ }^{1,2}$, Wendy Lee ${ }^{1,2}$, Dan Bandy ${ }^{1,2}$, Gene E. Alexander ${ }^{2,3}$, William J. Jagust ${ }^{4}$, Robert A. Koeppe ${ }^{5}$, Norman L. Foster ${ }^{6}$, Eric M. Reiman ${ }^{3,7},{ }^{1}$ Banner Alzheimer's Institute, Phoenix, AZ, USA; ${ }^{2}$ Arizona Alzheimer's Consortium, Phoenix, AZ, USA; ${ }^{3}$ University of Arizona, Tucson, AZ, USA; ${ }^{4}$ University of California, Berkeley, CA, USA; ${ }^{5}$ University of Michigan, Ann Arbor, MI, USA; ${ }^{6}$ University of Utah, Salt Lake City, UT, USA; ${ }^{7}$ Banner Alzheimer's Institute, Arizona Alzheimer's Consortium, Translational Genomics Research Institute, Phoenix, AZ, USA. Contact e-mail: Napatkamon.Ayutyanont@bannerhealth.com

Background: Alzheimer's disease (AD) is characterized by a characteristic pattern of reductions in the cerebral metabolic rate for glucose (CMRgl). We recently introduced use of an AD-Related Hypometabolism Overlap Index to determine the percentage of previously characterized AD-related hypometabolic brain voxels that are hypometabolic in an individual subject's fluorodeoxyglucose positron emission tomography (FDG-PET) image and help distinguish between probable $\mathrm{AD}$ patients, amnestic mild cognitive impairment (MCI) patients and normal controls (NC). The objective of sthis study is to evaluate the ability of our proposed Overlap Index to distinguish between probable $\mathrm{AD}$ patients, $\mathrm{MCI}$ patients who convert to probable $\mathrm{AD}$ within the next 18 months (MCIc), MCI patients who have so far remained stable (MCIs) and $\mathrm{NC}$ from the AD Neuroimaging Initiative (ADNI). Methods: The Overlap Index was computed in $74 \mathrm{AD}$ patients, $14 \mathrm{MCIc}, 124 \mathrm{MCIs}$ patients, and 82 NC as follows: First, statistical parametric mapping (SPM5) was used to generate individual cerebral hypometabolism z-score maps, comparing each person to a database of $82 \mathrm{NC}$. Next, the individual hypometabolism map was superimposed on an AD-related hypometabolic map generated from a previous study (Alexander et al, 2002). Finally, the Overlap Index was computed as $\mathrm{V}_{\mathrm{ADI}} / \mathrm{V}_{\mathrm{AD}} \times 100 \%$, such that $\mathrm{V}_{\mathrm{ADI}}$ is the hypometabolic volume in both previous AD-NC group comparison and the subsequent individual-NC comparison, and $\mathrm{V}_{\mathrm{AD}}$ is the hypometabolic volume in only the previous $\mathrm{AD}-\mathrm{NC}$ group comparison. Results: The Overlap Index significantly distinguished the four subject groups (ANOVA $\mathrm{P}=8.8 \mathrm{e}-15$ ). As predicted, the overlap index had the following rank order: ADMCIcMCIsNC (ANOVA with linear trend $\mathrm{P}=3.9 \mathrm{e}-16$ ). Moreover, the Overlap Index significantly distinguished between the MCIc group and the MCIs group (t-test $\mathrm{P}=0.013$ ). Conclusions: Our preliminary findings suggest that the AD Overlap Index can help identify hypometabolic patterns consistent with $\mathrm{AD}$ and help differentiate MCI patients converting to $\mathrm{AD}$ from stable MCI patients without the need to correct for multiple comparisons.

\section{P1-313 USE OF REGIONAL THICKNESS MEASURES TO PREDICT DECLINE IN QUESTIONABLE ALZHEIMER'S DISEASE DEMENTIA: COMPARISON TO STANDARD VOLUMETRIC MEASURES}

Akram Bakkour ${ }^{1}$, Bruce Fischl ${ }^{1}$, John C. Morris ${ }^{2}$, Randy L. Buckner ${ }^{3}$, Bradford C. Dickerson ${ }^{1},{ }^{1}$ Massachusetts General Hospital, Charlestown, MA, USA; ${ }^{2}$ Washington University, St. Louis, MO, USA; ${ }^{3}$ Harvard University, Cambridge, MA, USA. Contact e-mail: akram@nmr.mgh.harvard.edu

Background: We previously used exploratory analyses to determine that clinically mild Alzheimer's disease (AD) is reliably associated with a "cortical signature" of thinning in a specific set of regions in medial (MTL), inferior (ITG), and polar temporal (TP), inferior, superior (SPL), and medial parietal, and inferior and superior frontal cortices. We sought to determine whether the pattern of AD-related thinning is present in individuals with questionable dementia of the Alzheimer type (QAD) prior to mild $\mathrm{AD}$ and whether a greater degree of regional thinning predicts mild AD dementia. Methods: Participants included 49 older adults with QAD (Clinical Dementia Rating $(C D R)=0.5)$ at the time of structural MRI scanning. Nine regions of interest (ROIs) identified 